\title{
EVALUATION OF THE ROLE OF CEACAM1 IN THE DEVELOPING NEONATAL BRAIN AND ITS IMPACT ON ENCEPHALOPATHY OF PREMATURITY
}

\author{
S. Prager $^{1}$, B.B. Singer ${ }^{2}$, M. Keller ${ }^{1}$, I. Bendix ${ }^{1}$, G.W. Schlager ${ }^{1}$, M. Sifringer ${ }^{3}$, R. Herrmann ${ }^{1}$, H. Jastrow ${ }^{2}$, \\ S. Ergün ${ }^{2}$, U. Felderhoff-Müser ${ }^{1}$ \\ ${ }^{1}$ Department of Pediatrics 1, Neonatology, ${ }^{2}$ Department of Anatomy, University Hospital Essen, Essen, \\ ${ }^{3}$ Department of Anaesthesia and Intensive Care, Charité Berlin, Berlin, Germany
}

Encephalopathy of prematurity subsumes the two main neuropathological disorders of preterm white matter injury and neuronal/axonal disease. Factors such as hypoxia-ischemia, drug exposure, hyperoxia and maternal/neonatal inflammation are causal. Particularly with regards to infection/inflammation a significant role of CEACAM1 in different diseases is described. CEACAM1, a member of the carcinoembryonic antigen family of cell adhesion molecules, is a multifunctional molecule that contributes i.e. to morphogenesis of new blood vessels, cell proliferation, apoptosis, insulin metabolism, infection and inflammation. Because of its important role in inflammation-associated signaling we hypothesize that CEACAM1 might contribute to inflammation-induced perinatal brain injury. The aim of the current study is to evaluate the physiological regulation of CEACAM1 in the developing brain in newborn rats, which is yet unknown.

Brains of Wistar rats were obtained at day 1 to 15 . CEACAM1 protein expression was analysed in serial brain sections by immunohistochemistry including double labeling for markers such as MPB, GFAP, Neurofilament and Nestin to identify the CEACAM1-positive cell types using confocal microscopy. In addition immunostaining for CEACAM1 was performed on cultured pre-oligodendrocytes and mixed glia cells.

CEACAM1 expression was detected in broad areas of the brain beginning on day 5 in brain stem and afterwards in the forebrain. CEACAM1 immunostaining on cultivated cells revealed its expression in oligodendrocytes.

Our results show for the first time an expression of CEACAM1 in oligodendrocytes. This is of special importance since white matter injury is a myelination disorder. Alterations of CEACAM1 expression under pathologic conditions are subject of current investigations. 